\title{
Modelling infectious diseases with herd immunity in a randomly mixed population
}

Kian Boon Law ( $\nabla$ kblaw@crc.gov.my )

Institute for Clinical Research

Kalaiarasu M. Peariasamy

Institute for Clinical Research

Hishamshah Mohd. Ibrahim

Ministry of Health Malaysia

Noor Hisham Abdullah

Ministry of Health Malaysia

\section{Research Article}

Keywords: Herd immunity, Deterministic model, Vaccine, Vaccination, Population immunity, Herd immunity threshold

Posted Date: April 14th, 2021

DOI: https://doi.org/10.21203/rs.3.rs-289776/v6

License: (1) (1) This work is licensed under a Creative Commons Attribution 4.0 International License.

Read Full License 
1

2

3

4

5

\section{Modelling infectious diseases with herd immunity in a randomly mixed population}

Kian Boon Law ${ }^{1 *}$, Kalaiarasu M. Peariasamy ${ }^{1}$, Hishamshah Ibrahim $^{2}$, Noor Hisham Abdullah ${ }^{2}$

${ }^{1}$ Institute for Clinical Research, National Institutes of Health, Ministry of Health Malaysia, Setia Alam, Malaysia

2 The Office of Director General, Ministry of Health Malaysia, Putrajaya, Malaysia

\section{*Corresponding author:}

Kian Boon, Law

Email: kblaw@crc.gov.my, telephone details:+6012 7040414 


\section{Abstract}

\section{Background}

The conventional susceptible-infectious-recovered (SIR) model tends to overestimate the transmission dynamics of infectious diseases and ends up with total infections and total immunized population exceeding the threshold required for control and eradication of infectious diseases. The study aims to overcome the limitation by allowing the transmission rate of

\section{Methods}

Two new SIR models were developed to mimic the declining transmission rate of infectious diseases at different stages of transmission. Model A mimicked the declining transmission rate along with the reducing risk of transmission following infection, while Model B mimicked the declining transmission rate following recovery. Then, the conventional SIR model, Model A and Model B were used to simulate an infectious disease with a basic reproduction number $\left(\mathrm{r}_{0}\right)$ of 3.0 and a herd immunity threshold (HIT) of 0.667 with and without vaccination. The infectious disease was expected to be controlled or eradicated when the total immunized population either through infection or vaccination reached the level predicted by the HIT. Outcomes of simulations were assessed at the time when the total immunized population reached the level predicted by the HIT, and at the end of simulations.
\end{abstract}

\title{
Findings
}

All three models performed likewise at the beginning of the transmission when sizes of infectious and recovered were relatively small as compared with the population size. The infectious disease modelled using the conventional SIR model appeared completely out of control even when the HIT was achieved in all scenarios with and without vaccination. The infectious disease modelled using Model A appeared to be controlled at the level predicted by the HIT in all scenarios with and without vaccination. Model B projected the infectious disease to be controlled at the level predicted by the HIT only at high vaccination rates. At lower vaccination rates or without vaccination, the level at which the infectious disease was controlled cannot be accurately predicted by the HIT.

\section{Conclusion}

Transmission dynamics of infectious diseases with herd immunity can accurately be modelled by allowing the transmission rate of infectious disease to decline along with the combined risk of contact infection. Model B provides a more credible framework for modelling infectious diseases with herd immunity in a randomly mixed population.

\section{Funding \\ No funding sources}

\section{Keywords}

Herd immunity; Deterministic model; Vaccine; Vaccination; Population immunity; Herd immunity threshold 


\section{Introduction}

Herd or population immunity refers to the indirect protection from infectious diseases among remaining susceptible individuals when most people in a population are immune to infectious diseases either through vaccination or infection. The concept of herd immunity became a fixture of epidemiology in 1930s, and took on fresh prominence in 1950s and 1960s as new vaccines raised crucial questions for public health policy on the proportion of the vaccinated population for the eradication of infectious diseases ${ }^{1}$. Herd immunity takes effect when the transmission rate of infectious diseases starts to decline along with the reducing risk of infection due to the presence or proximity of immune individuals in a randomly mixed population ${ }^{2}$. Although the effect of herd immunity has been observed in many vaccinated populations of periodical childhood epidemics, such as measles, mumps, rubella, pertussis, chickenpox and polio, it has not been successfully attained through mathematical modelling.

Mathematical models such as the susceptible-infectious-recovered (SIR) and its variants are widely used to simulate the transmission pattern of infectious diseases. Those models use a flexible compartmental framework with robust assumptions for a wide range of applications ${ }^{3-6}$. The compartmental framework of SIR model simplifies the transmission dynamics of infectious diseases by classifying individuals based on their epidemiological status and ability to host and transmit a pathogens ${ }^{7}$. Other than the framework, the SIR model also assumes complete immunity can be acquired through infection, hence encompassing the epidemiological notion of herd immunity through infection ${ }^{8,9}$.

It is generally believed that infectious diseases can be controlled or eradicated when the total immunized population reaches a level predicted by the herd immunity threshold (HIT). The HIT indicates a level of one infected individual generating less than one secondary case on average $^{10}$. The transmission of infectious diseases becomes unsustainable in a population beyond the HIT. The HIT can be calculated from the basic reproduction number $\left(\mathrm{r}_{0}\right)$ of infectious diseases to guide the vaccination strategy for controlling an epidemic or pandemic, either through vaccine or infection ${ }^{2,11}$. For instance, to control or eradicate the COVID-19 pandemic, vaccination should cover $50.0 \%$ to $66.7 \%$ of the world population based on the ro of 2.0 to 3.0 for the novel coronavirus ${ }^{12-15}$.

The conventional SIR model often overestimates the transmission dynamics of infectious diseases. For instance, an infectious disease with $\mathrm{r}_{0}$ of 3.0 would eventually infect up to $94.0 \%$ of a population, a level way beyond the expected HIT, even with the presence of herd immunity if modelled using the conventional SIR model. This problem is of great concern especially for time being as many models have been developed based on the conventional SIR framework to guide public health planning and preparedness against the COVID-19 pandemic ${ }^{16-19}$. This study aims to investigate and overcome the aforesaid limitation of using the SIR model in modelling infectious diseases with herd immunity in a randomly mixed population. We proposed a key modification to the conventional SIR model that allows the transmission rate of infectious disease to decline along with the reducing risk of contact infection, which is more in line with the principle of herd immunity. 


\section{Methods}

\section{Conventional SIR model}

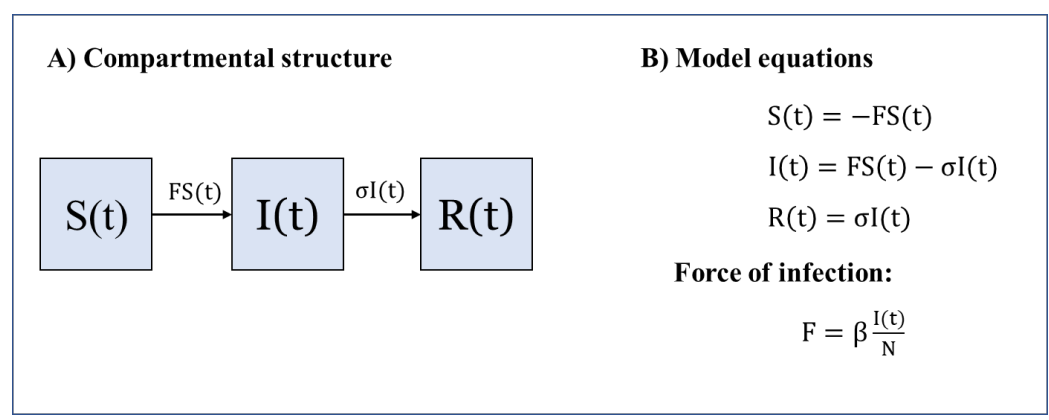

Figure 1: The compartmental structure and model equations of Kermack \& Mckendrick's SIR model.

Kermack \& McKendrick postulated the first SIR model for infectious diseases in 1927 before vaccines became popular in 1950s for control and eradication of infectious diseases ${ }^{9}$. Then, the SIR model became the fundamental of most infectious disease models developed. The conventional SIR model divides a homogenous population, $\mathrm{N}$ into three basic compartments: susceptible denoted by $\mathrm{S}(\mathrm{t})$, infectious denoted by $\mathrm{I}(\mathrm{t})$, and recovered or removed denoted by $\mathrm{R}(\mathrm{t})$, and assumes infectious diseases spread from affected to unaffected through contact infection (Fig.1). Susceptible are individuals who have an equal risk of being infected. Infectious are individuals who have developed infectivity and can transmit pathogen to those who remain susceptible. Recovered or removed are individuals who have recovered from infection and immune to reinfection. In brief, the conventional SIR model describes the transmission of infectious disease with herd immunity through infection. The SIR model can be described mathematically by a set of ordinary differential equations (ODEs) as shown in Fig. 1.

According to model equations (Fig. 1), the rate of individuals moving from compartment $S(t)$ to $I(t)$ due to contact infection is determined by $S(t)$ and the force of infection, $F$, which consists of the product of a constant contact rate $(\beta)$ and the proportion of infectious individuals, $\frac{I(t)}{N}$. And, the rate of individuals moving from compartment $I(t)$ and $R(t)$ following recovery is determined by $\mathrm{I}(\mathrm{t})$ and the reciprocal of infection duration, denoted by $\sigma$. Therefore, the conventional SIR model often simulates the $\mathrm{I}(\mathrm{t})$ to increase at the beginning of transmission and subsequently diminishes due to the exhausting stock of $S(t)$.

Without vital dynamics, the population size is constant and can be given by:

$$
\mathrm{N}=\mathrm{S}(\mathrm{t})+\mathrm{I}(\mathrm{t})+\mathrm{R}(\mathrm{t})
$$

Equation (1) can be converted into prevalence or proportion by dividing each notation with the population size, $\mathrm{N}$ :

$$
1=\frac{S(t)}{N}+\frac{I(t)}{N}+\frac{R(t)}{N}
$$


According to Equation (2), $\mathrm{I}(\mathrm{t})$ and $\mathrm{R}(\mathrm{t})$ are often very small as compared with $\mathrm{N}$ at the

177 beginning of the transmission, therefore, $\frac{\mathrm{I}(\mathrm{t})}{\mathrm{N}} \approx 0, \frac{\mathrm{R}(\mathrm{t})}{\mathrm{N}} \approx 0$, and $\frac{\mathrm{S}(\mathrm{t})}{\mathrm{N}} \approx 1$. At the end of

178 transmission, the $\mathrm{I}(\mathrm{t})$ would become very small again, therefore, $1-\frac{\mathrm{R}(\mathrm{t})}{\mathrm{N}} \approx \frac{\mathrm{S}(\mathrm{t})}{\mathrm{N}}$. To model

179 infectious diseases with herd immunity, $\frac{S(t)}{N}$ or $1-\frac{R(t)}{N}$ can be incorporated into the force of

180 infection to mimic the reducing risk of contact infection at different stages of infection.

181

182

By incorporating $\frac{\mathrm{S}(\mathrm{t})}{\mathrm{N}}$ into the $\mathrm{F}$, we assume herd immunity takes effect to reduce the transmission rate of infectious diseases along with the reducing risk of contact infection following infection. By incorporating $1-\frac{\mathrm{R}(\mathrm{t})}{\mathrm{N}}$ into the $\mathrm{F}$, we assume herd immunity takes effect to reduce the transmission rate of infectious diseases along with the reducing risk of contact infection following recovery.

\section{New SIR models}

191

In chemistry, the Law of Mass Action is used to describe the rate of chemical reactions being proportional to the concentration of reactants. ${ }^{20}$ Based on the above principle, a contact can be regarded as an interactive event between susceptible individuals and infectious individuals in a randomly mixed environment, with its rate being proportional to $\frac{S(t)}{N}$ and $\frac{I(t)}{N}$. The product of $\frac{\mathrm{S}(\mathrm{t})}{\mathrm{N}}$ and $\frac{\mathrm{I}(\mathrm{t})}{\mathrm{N}}$ denotes the combined risk of contact infection. Two new models were developed 195 based on the above principle as below:

Model A: The total transmission rate of infectious diseases in a randomly mixed population depends on the $\mathrm{S}(\mathrm{t})$ and a new force of infection, $\mathrm{F}_{\mathrm{A}}$, which consists of the product of $\beta, \frac{\mathrm{I}(\mathrm{t})}{\mathrm{N}}$ and $\frac{\mathrm{S}(\mathrm{t})}{\mathrm{N}}$ as follow:

$$
F_{A}=\beta \frac{I(t) S(t)}{N^{2}}
$$

In Model A, the risk of contact infection is determined by both $\frac{S(t)}{N}$ and $\frac{I(t)}{N}$. Therefore, the transmission rate would decline along with the reducing risk of contact infection when individuals move from compartment $\mathrm{S}(\mathrm{t})$ to $\mathrm{I}(\mathrm{t})$ due to infection. The compartmental structure and model equations of Model A can be found in Fig. 2.

Model B: The total transmission rate of infectious diseases in a randomly mixed population depends on the $\mathrm{S}(\mathrm{t})$ and a new force of infection, $\mathrm{F}_{\mathrm{B}}$, which consists of the product of $\beta, \frac{\mathrm{I}(\mathrm{t})}{\mathrm{N}}$ and $1-\frac{\mathrm{R}(\mathrm{t})}{\mathrm{N}}$ as follow:

$$
\mathrm{F}_{\mathrm{B}}=\beta \frac{\mathrm{I}(\mathrm{t})[\mathrm{N}-\mathrm{R}(\mathrm{t})]}{\mathrm{N}^{2}} .
$$


214 In Model B, the risk of contact infection is determined by both $1-\frac{R(t)}{N}$ or $\frac{N-R(t)}{N}$ and $\frac{I(t)}{N}$.

215 Therefore, the transmission rate would decline along with the reducing risk of contact infection 216 when individuals move from compartment $\mathrm{I}(\mathrm{t})$ to $\mathrm{R}(\mathrm{t})$ after recovery. The $1-\frac{\mathrm{R}(\mathrm{t})}{\mathrm{N}}$ denotes the 217 inverse of proportion of recovered individuals and is used to mimic the reducing risk of contact 218 infection following recovery. The compartmental structure and model equations of Model B can 219 be found in Fig. 2.

A) Compartmental structure

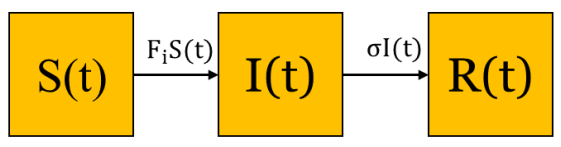

B) Model equations

$$
\begin{aligned}
& S(t)=-F_{i} S(t) \\
& I(t)=F_{i} S(t)-\sigma I(t) \\
& R(t)=\sigma I(t)
\end{aligned}
$$

Force of infection:

Model A: $F_{i=A}=\beta \frac{I(t)[S(t)]^{2}}{N^{2}}$

Model B: $F_{i=B}=\beta \frac{I(t)[N-R(t)]}{N^{2}}$
Figure 2: The

compartmental structure and model equations of the newly developed Model A and Model B.

Both Model A and Model B retain the basic SIR compartmental structure, except for the force of infection (Fig. 2). With the modification, both models can be used to simulate the transmission dynamics of infectious diseases with herd immunity through infection in a randomly mixed population.

\section{The basic reproduction number, $\mathbf{r}_{0}$}

The equation of I(t) from the conventional SIR model, Model A and Model B can be rearranged as follows:

$$
\frac{d I(t)}{d t}=\left[\beta \frac{S(t)}{N}-\sigma\right] I(t)
$$

$$
\frac{\mathrm{dI}(\mathrm{t})}{\mathrm{dt}}=\left[\beta\left(\frac{\mathrm{S}(\mathrm{t})}{\mathrm{N}}\right)^{2}-\sigma\right] \mathrm{I}(\mathrm{t}) \text {. }
$$

$$
\frac{\mathrm{d}(\mathrm{t})}{\mathrm{dt}}=\left[\beta\left(\frac{\mathrm{S}(\mathrm{t})[\mathrm{N}-\mathrm{R}(\mathrm{t})]}{\mathrm{N}^{2}}\right)-\sigma\right] \mathrm{I}(\mathrm{t}) .
$$

238

239

At the beginning of the transmission, when $\frac{\mathrm{S}(\mathrm{t})}{\mathrm{N}} \approx 1$ and $\frac{\mathrm{R}(\mathrm{t})}{\mathrm{N}} \approx 0$, we would obtain the exact equation for all three models as follow:

$$
\frac{d I(t)}{d t}=(\beta-\sigma) I(t)
$$

The integral of Equation (8) is an exponential function as follow: 


$$
\mathrm{I}(\mathrm{t})=\mathrm{I}_{0} \mathrm{e}^{(\beta-\sigma) \mathrm{t}}
$$

Equation (9) shows a crucial condition that determines the widespread of infectious diseases in a population. The transmission of infectious diseases can be sustained if $\beta>\sigma$ or $\frac{\beta}{\sigma}>$ 1. The ratio between $\beta$ and $\sigma$ denotes the basic reproduction number $\left(\mathrm{r}_{0}\right)$ of infectious diseases. The ro can also be defined as the number of secondary cases caused by a single primary case in a wholly susceptible population ${ }^{21,22}$. The ro can be used to derive the herd immunity threshold (HIT) according to a simple theorem proposed by Dietz $(1975)^{23}$.

$$
\mathrm{HIT}=1-\frac{1}{\mathrm{r}_{0}} .
$$

The HIT can also be defined as the level that the transmission of infectious diseases becomes unsustainable when one infected person generates less than one secondary case on average in a population ${ }^{10}$. Often, the HIT can be used to predict total infections achieved at the end of transmission without vaccination. If vaccination is used to control infectious diseases, the HIT indicates the share of a population that needs to be vaccinated for control of infectious diseases.

\section{Vaccine models}

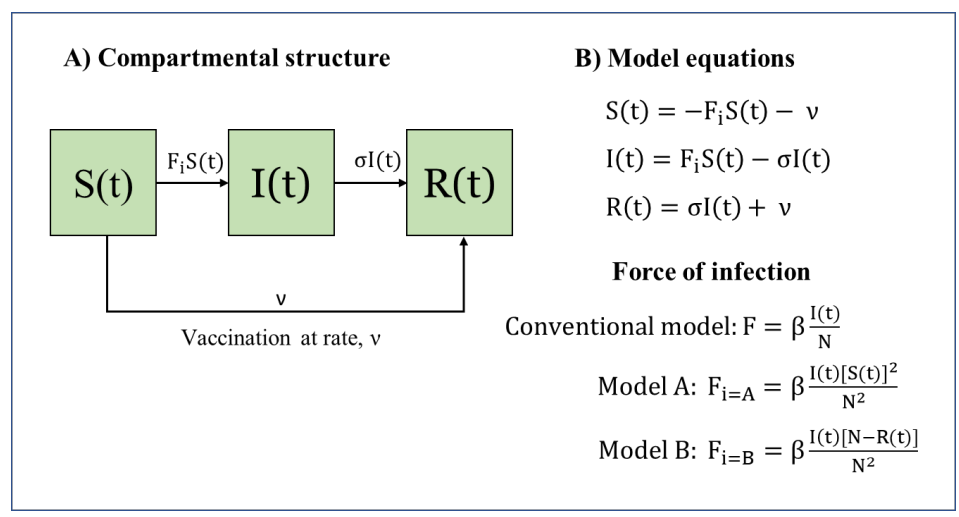

Figure 3: The

compartmental structure and model equations of vaccine models modified using the conventional SIR model, Model A and Model B.

Unlike immunity through infection, vaccination introduces immunity into individuals without developing infectivity, therefore protecting a population from infectious diseases without generating more infections. A simple vaccine model can be created using the conventional SIR model, Model A and Model B by allowing individuals vaccinated to move from compartment $\mathrm{S}(\mathrm{t})$ straight to $\mathrm{R}(\mathrm{t})$ at a constant vaccination rate denoted by $v$ (Fig. 3 ). The magnitude of $v$ depends on factors such as the availability of vaccines and resources allocated for vaccinating people, not the size of $\mathrm{S}(\mathrm{t})$. Here, we assume individuals who have been vaccinated would develop complete immunity as those developing immunity through infection. Therefore, the compartment $\mathrm{R}(\mathrm{t})$ would consist of the total immunized population either through infection or vaccination. Herd immunity was considered achieved when the total immunized population reached the level predicted by the HIT.

Table 1 shows the breakdown of transmission rate and recovery rate of the conventional SIR model, Model A and Model B. Model A and Model B only differ from the conventional SIR 
model in the risk of contact infection. By assigning the same value to $\beta, \sigma$ and $v$, all three models can be used to simulate the transmission dynamics of the same infectious disease with herd immunity either through infection or vaccination in a randomly mixed population.

Table 1: Breakdown of transmission rate and recovery rate of the conventional SIR model, Model A and Model B.

\begin{tabular}{|c|c|c|c|c|c|c|c|c|}
\hline \multirow{3}{*}{ Models } & \multicolumn{3}{|c|}{ Components of transmission rate } & \multirow{3}{*}{$\begin{array}{l}\text { Total } \\
\text { transmission } \\
\text { rate from } S(t) \\
\text { to } I(t)\end{array}$} & \multirow{2}{*}{\multicolumn{2}{|c|}{$\begin{array}{l}\text { Components of } \\
\text { recovery rate }\end{array}$}} & \multirow{3}{*}{$\begin{array}{l}\text { Total } \\
\text { recovery } \\
\text { rate from } \\
\mathrm{I}(\mathrm{t}) \text { to } \mathrm{R}(\mathrm{t})\end{array}$} & \multirow{3}{*}{$\begin{array}{l}\text { Vaccination } \\
\text { rate }\end{array}$} \\
\hline & \multicolumn{2}{|c|}{ Force of infection, F } & \multirow[b]{2}{*}{$\begin{array}{l}\text { Number of } \\
\text { Susceptible }\end{array}$} & & & & & \\
\hline & $\begin{array}{l}\text { Contact } \\
\text { rate }\end{array}$ & $\begin{array}{c}\text { Risk of } \\
\text { contact } \\
\text { infection } \\
\end{array}$ & & & $\begin{array}{l}\text { Recovery } \\
\text { coefficient }\end{array}$ & $\begin{array}{l}\text { Number of } \\
\text { Infectious }\end{array}$ & & \\
\hline $\begin{array}{l}\text { Conventional } \\
\text { SIR model }\end{array}$ & $\beta$ & $\frac{I(t)}{N}$ & $S(t)$ & $\beta \frac{\mathrm{I}(\mathrm{t}) \mathrm{S}(\mathrm{t})}{\mathrm{N}}$ & $\sigma$ & $\mathrm{I}(\mathrm{t})$ & $\sigma \mathrm{I}(\mathrm{t})$ & $v$ \\
\hline Model A & $\beta$ & $\frac{\mathrm{I}(\mathrm{t}) \mathrm{S}(\mathrm{t})}{\mathrm{N}^{2}}$ & $\mathrm{~S}(\mathrm{t})$ & $\beta \frac{I(t)[S(t)]^{2}}{N^{2}}$ & $\sigma$ & $\mathrm{I}(\mathrm{t})$ & $\sigma \mathrm{I}(\mathrm{t})$ & $v$ \\
\hline Model B & $\beta$ & $\frac{\mathrm{I}(\mathrm{t})[\mathrm{N}-\mathrm{R}(\mathrm{t})]}{\mathrm{N}^{2}}$ & $\mathrm{~S}(\mathrm{t})$ & $\beta \frac{\mathrm{I}(\mathrm{t}) \mathrm{S}(\mathrm{t})[\mathrm{N}-\mathrm{R}(\mathrm{t})]}{\mathrm{N}^{2}}$ & $\sigma$ & $\mathrm{I}(\mathrm{t})$ & $\sigma \mathrm{I}(\mathrm{t})$ & $v$ \\
\hline
\end{tabular}

\section{Simulations and sensitivity analyses}

Model ODEs of all three models can be solved by using numerical integration. First, we simulated all three models under the exact and arbitrary conditions with parameter values as presented in Table 2. These parameter values allowed all three models to project the transmission dynamics of the same infectious disease in a homogenous population. We assumed complete immunity can be acquired either through infection or vaccination, therefore, herd immunity can be developed either through infection or vaccination. Herd immunity was considered achieved when the total immunized population reached the level predicted by the HIT.

Transmission dynamics of the infectious disease with herd immunity through infection only were simulated using models as presented in Fig. 1 and Fig. 2. We expected the infectious disease to subside when the total immunized population or $\mathrm{R}(\mathrm{t})$ reached the level predicted by the HIT. We evaluated the size of each compartment at the time when the HIT was reached, and at the end of simulation $(\mathrm{t}=200)$.

Transmission dynamics of the infectious disease with herd immunity through vaccination and infection were simulated using models as presented in Fig. 3. The total immunized population would consist of those who had developed immunity through infection or vaccination. At high vaccination rates, the total immunized population was largely contributed by those acquiring immunity through vaccination. At low vaccination rates, the total immunized population was largely contributed by those acquiring immunity through infection. We simulated all vaccine models at three vaccination rates, as stated in Table 2 . At all vaccination rates, we evaluated the size of each compartment at the time when the HIT was reached, and at the end of simulations ( $\mathrm{t}=500$ for $v=1.0 \%, \mathrm{t}=300$ for $\nu=0.5 \%$, and $\mathrm{t}=200$ for $v=0.1 \%$ ). The vaccination rate was set to zero after the HIT was reached until the end of simulations. 
Table 2: Parameter values used in simulations and sensitivity analyses

\begin{tabular}{|l|l|}
\hline Parameters & Values \\
\hline Contact rate, $\beta$ & 0.3 \\
& 0.11 to 0.4 (Sensitivity analysis) \\
\hline Recovery coefficient, $\sigma$ & 0.1 \\
\hline Infection duration & 10 \\
\hline Basic reproduction number, $\mathrm{r}_{0}$ & 3.0 \\
& 1.1 to $4.0($ Sensitivity analysis) \\
\hline Herd immunity threshold, HIT & $0.667(66.7 \%)$ \\
\hline Vaccination rate, $v$ & $1.0 \%$ population per unit $\mathrm{t}$ \\
& $0.5 \%$ population per unit $\mathrm{t}$ \\
& $0.1 \%$ population per unit $\mathrm{t}$ \\
\hline Population size, $\mathrm{N}$ & 1000000 \\
\hline Initial value for $\mathrm{I}(\mathrm{t})$ & 1 \\
\hline Initial value for $\mathrm{S}(\mathrm{t})$ & $\mathrm{N}-\mathrm{I}(\mathrm{t})$ \\
\hline Initial value for $\mathrm{R}(\mathrm{t})$ & 0 \\
\hline Initial value for Total infections & 1 \\
\hline
\end{tabular}

\section{Results:}

\section{Transmission dynamics of infectious diseases with herd immunity through infection}

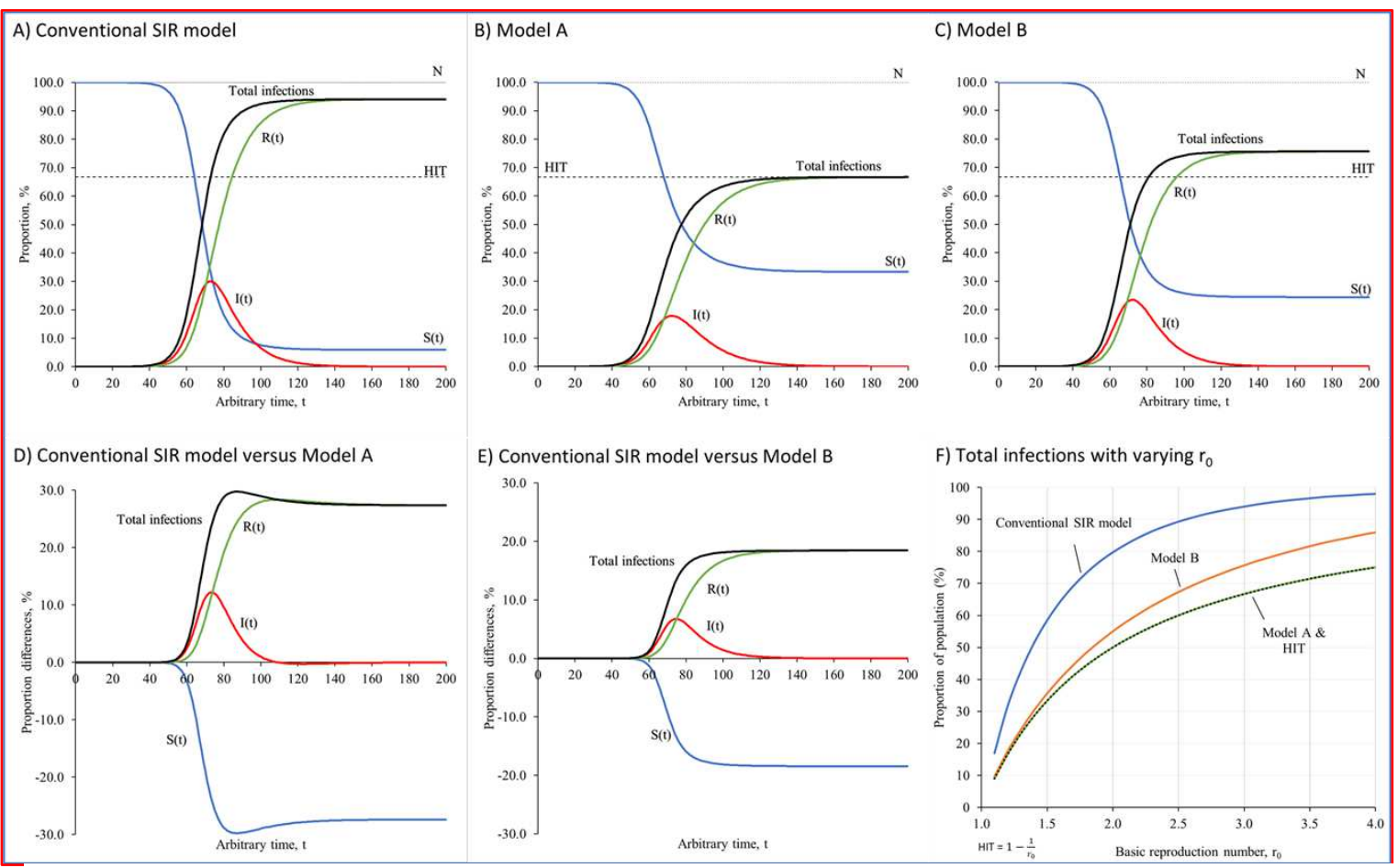

Figure 4: Transmission dynamics of infectious diseases with herd immunity through infection

Part A, B and C presents transmission dynamics of infectious diseases with herd immunity through infection simulated by the conventional SIR model, Model A and Model B. Part D and E presents proportion differences between the conventional SIR model and Model A and between the conventional SIR mode and Model B. Part F presents total infections generated by the conventional SIR model, Model A and Model B with varying $\mathrm{r}_{0}$. 
Without vaccination, all three models described the transmission dynamics of infectious diseases with herd immunity through infection as individuals who had been infected were assumed to recover with complete immunity. Figure 4 presents the transmission dynamics of infectious disease with $\mathrm{r}_{0}$ of 3.0 simulated using the conventional SIR model, Model A and Model B. Our simulations showed that all three models performed likewise at the beginning of the transmission when both $\mathrm{I}(\mathrm{t})$ and $\mathrm{R}(\mathrm{t})$ were relatively small as compared with the population size, N.

According to the conventional SIR model, the total $\mathrm{R}(\mathrm{t})$ or immunized population through infection would reach the level predicted by the HIT at $\mathrm{t}=134$, with total infections reaching $86.9 \%, \mathrm{I}(\mathrm{t})$ reaching $19.2 \%$ and $\mathrm{S}(\mathrm{t})$ reaching $13.1 \%$. After that, the infectious disease would continue to infect more people, and ended up with up to $94.0 \%$ of population being infected, leaving only $6.0 \%$ of population remaining susceptible (Fig. 4A and Table 3 ). Model A projected the infectious disease to be controlled and eradicated at the level accurately predicted by the HIT at $\mathrm{t}=195$ until the end of simulation (Fig. 4B and Table 3). Model B projected the infectious disease to subside at a level higher than the HIT, with total infections reaching 75.6\%, leaving $24.4 \%$ of population to remain susceptible in the population (Fig. $4 \mathrm{C}$ and Table 3 ).

According to our simulations, the transmission of infectious disease started to be controlled and suppressed after $\mathrm{t}=50$ in both Model A and Model B as compared with the conventional SIR model (Fig. 4D and 4E). Our sensitivity analysis shows that total infections generated by the conventional SIR model at the end of simulation were completely way above the level predicted by the HIT across all ro values. Total infections generated by Model A at the end of simulation were accurately predicted by the HIT across all $\mathrm{r}_{0}$ values, while total infections generated by Model B at the end of simulation were predicted the HIT when $\mathrm{r}_{0}$ was small and deviated away from the HIT at higher $\mathrm{r}_{0}$ values in Model B (Fig. 4F).

By allowing the transmission rate to decline along with the reducing risk of contact infection, Model A simulated the infectious disease to subside at a level predicted by the $\mathrm{r}_{0}$ and HIT accurately. However, it might be too ideal to assume that the risk of contact infection and transmission rate can be reduced by herd immunity immediately following infection, unless in the context of high vaccination rates. Model B was more in line with the fundamental of herd immunity. It was more realistic to allow the transmission rate to decline along with the reducing risk of contact infection following recovery.

\section{Transmission dynamics of infectious diseases with herd immunity through vaccination}

With modification as shown in Fig.3, all three models can be used to describe the transmission dynamics of infectious diseases with herd immunity through vaccination. At a very high vaccination rate $(v=1.0 \%$ population per unit $t)$, our simulations showed that total infections would continue to increase at a lower rate even after the HIT was achieved in the conventional SIR model (Fig. 5A). The conventional SIR model failed to demonstrate either control or eradication of infectious diseases even at a high vaccination rate after the HIT was achieved, let alone lower vaccination rates. On the other hand, both Model A and Model B projected the infectious disease to be controlled and eradicated when the total immunized population reached 
the level predicted by the HIT. At a very high vaccination rate, both Model A and Model B performed likewise and generated the same outcome with total infections controlled at $0.02 \%$ after the HIT was achieved at $\mathrm{t}=67$ until the end of the simulation (Fig. 5B and 5C, Table 3).

At a lower vaccination rate $(v=0.5 \%$ population per unit $t)$, total infections continued to increase at a higher rate even after the HIT was achieved at $\mathrm{t}=100$ in the conventional SIR model (Fig. 5D, Table 3). At $\mathrm{t}=100$, the total immunized population reached $67.58 \%$ of population, consisting of $18.08 \%$ of population immunized through infection and $49.50 \%$ of population immunized through vaccination. The infectious disease appeared completely out of control and continued to infect more people in the population, causing total infections to increase by another $8.06 \%$ and reached $34.37 \%$ at the end of simulation $(t=500)$. Both Model A and Model B continued to project the infectious disease to be controlled even at a lower vaccination rate. At $v=0.5 \%$ population per unit $t$, the total immunized population would reach the level predicted by the HIT at $\mathrm{t}=131$ in both models, and total infections were controlled at $1.22 \%$ to $1.24 \%$ in Model A and $1.24 \%$ to $1.26 \%$ in Model B, respectively (Fig. 5E and 5F, Table 3).

At the lowest vaccination rate $(v=0.1 \%$ population per unit $t)$, the herd immunity was largely contributed by those immunized through infection. The total immunized population would reach the level predicted by the HIT at $\mathrm{t}=87$, with total infections of $76.33 \%$ in the conventional SIR model. Subsequently, total infections continued to increase by $8.09 \%$ to reach $84.42 \%$ at the end of simulation (Fig. 5G, Table 3). As for Model A, the total immunized population would reach the level predicted by the HIT at $\mathrm{t}=134$, with total infections of $54.08 \%$. At the end of simulation, total infections only increased by another $0.36 \%$ to $54.44 \%$ in Model A (Fig. 5H, Table 3). In model B, the HIT was reached at $\mathrm{t}=107$, with total infections of $60.68 \%$. At the end of simulation, total infections continued to increase only by another $1.44 \%$ in Model B (Fig. 5I, Table 3). 

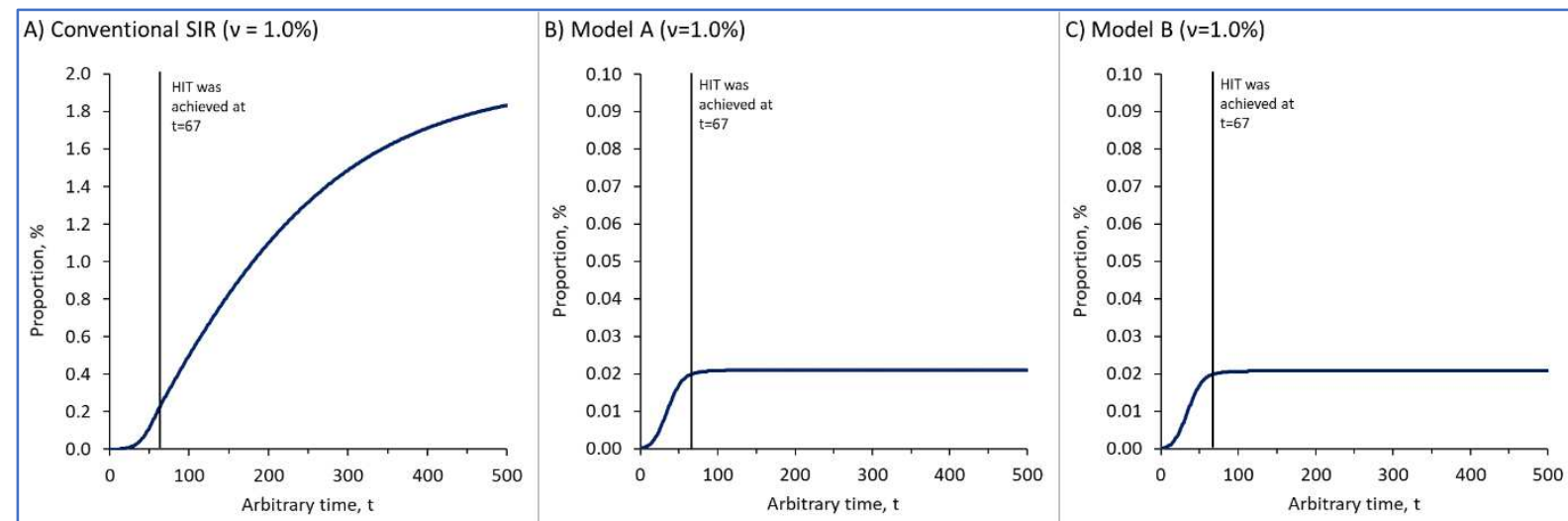

D) Conventional SIR ( $v=0.5 \%$ )

E) Model A ( $v=0.5 \%)$

F) Model $B(v=0.5 \%)$
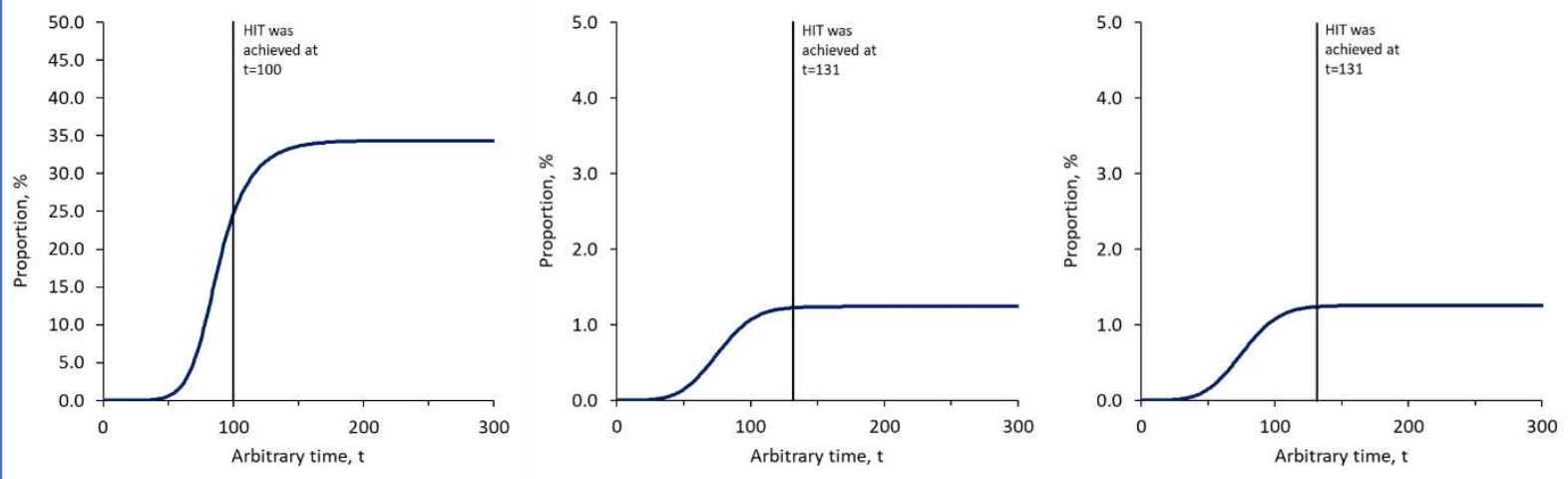

G) Conventional SIR ( $v=0.1 \%)$

H) Model A ( $v=0.1 \%)$

1) Model B ( $v=0.1 \%)$
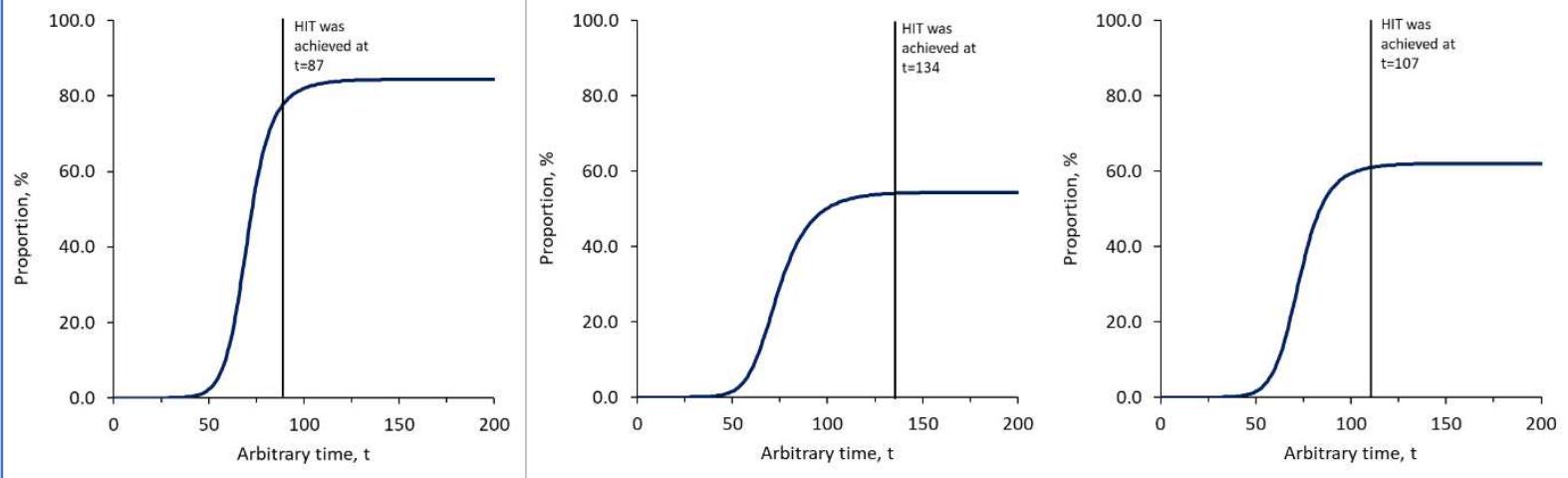

Figure 5: Transmission dynamics of infectious diseases with herd immunity through vaccination

Part A, B and C present transmission dynamics of infectious diseases at vaccination rate, $v=1.0 \%$ population per unit $\mathrm{t}$ projected by the conventional SIR model, Model A and Model B. The HIT was reached at $\mathrm{t}=67$ in all three models. Part D, $\mathrm{E}$ and $\mathrm{F}$ present transmission dynamics of infectious diseases at $v=0.5 \%$ population per unit $\mathrm{t}$ projected by the conventional SIR model, Model A and Model B. The HIT was reached at $\mathrm{t}=100$ in the conventional SIR model, and at $\mathrm{t}=131$ in both Model A and Model B. Part G, $\mathrm{H}$ and I present transmission dynamics of infectious disease at $v=0.1 \%$ population per unit $\mathrm{t}$ projected by the conventional SIR model, Model A and Model B. The HIT was reached at $\mathrm{t}=87$ in the conventional SIR 
Table 3: Outputs of simulations using the conventional SIR model, Model A and Model B.

\begin{tabular}{|c|c|c|c|}
\hline Outputs & $\begin{array}{c}\begin{array}{c}\text { Conventional SIR } \\
\text { model }\end{array} \\
\end{array}$ & Model A & Model B \\
\hline \multicolumn{4}{|c|}{ Herd immunity through infection } \\
\hline HIT was achieved at: & $\mathrm{t}=134$ & $\mathrm{t}=195$ & $\mathrm{t}=97$ \\
\hline$-\mathrm{S}(\mathrm{t})$ & 13.1 & 33.3 & 26.2 \\
\hline$-\mathrm{I}(\mathrm{t})$ & 19.2 & 0 & 6.5 \\
\hline - Total infections & 86.9 & 66.7 & 73.8 \\
\hline$-\mathrm{R}(\mathrm{t})$ & 67.7 & 66.7 & 67.3 \\
\hline \multicolumn{4}{|l|}{ At the end of simulation $(\mathrm{t}=200)$} \\
\hline$-\mathrm{S}(\mathrm{t})$ & 6.0 & 33.3 & 24.4 \\
\hline - Total infections & 94.0 & 66.7 & 75.6 \\
\hline$-\mathrm{R}(\mathrm{t})$ & 94.0 & 66.7 & 75.6 \\
\hline \multicolumn{4}{|c|}{ Herd immunity through vaccination } \\
\hline \multicolumn{4}{|c|}{ A) $v=1.0 \%$ population per unit t } \\
\hline HIT was achieved at: & $\mathrm{t}=67$ & $\mathrm{t}=67$ & $\mathrm{t}=67$ \\
\hline$-\mathrm{S}(\mathrm{t})$ & 32.74 & 32.98 & 32.98 \\
\hline$-\mathrm{I}(\mathrm{t})$ & 0.08 & 0 & 0 \\
\hline - Total infections & 0.26 & 0.02 & 0.02 \\
\hline - Total immunized & 67.18 & 67.02 & 67.02 \\
\hline - Through infection & 0.18 & 0.02 & 0.02 \\
\hline - Through vaccination & 67.00 & 67.00 & 67.00 \\
\hline \multicolumn{4}{|l|}{ At the end of simulation $(t=500)$} \\
\hline$-\mathrm{S}(\mathrm{t})$ & 31.17 & 32.98 & 32.98 \\
\hline - Total infections & 1.83 & 0.02 & 0.02 \\
\hline - Total immunized & 68.82 & 67.02 & 67.02 \\
\hline - Through infection & 1.82 & 0.02 & 0.02 \\
\hline - Through vaccination & 67.00 & 67.00 & 67.00 \\
\hline \multicolumn{4}{|c|}{ B) $v=0.5 \%$ population per unit t } \\
\hline HIT was achieved at: & $\mathrm{t}=100$ & $\mathrm{t}=131$ & $\mathrm{t}=131$ \\
\hline$-\mathrm{S}(\mathrm{t})$ & 26.31 & 33.28 & 33.26 \\
\hline$-\mathrm{I}(\mathrm{t})$ & 6.12 & 0.04 & 0.04 \\
\hline - Total infections & 24.19 & 1.22 & 1.24 \\
\hline - Total immunized & 67.58 & 66.69 & 66.70 \\
\hline - Through infection & 18.08 & 1.19 & 1.20 \\
\hline - Through vaccination & 49.50 & 65.50 & 65.50 \\
\hline \multicolumn{4}{|l|}{ At the end of simulation $(t=300)$} \\
\hline$-\mathrm{S}(\mathrm{t})$ & 16.14 & 33.26 & 33.24 \\
\hline - Total infections & 34.37 & 1.24 & 1.26 \\
\hline - Total immunized & 83.86 & 66.74 & 66.76 \\
\hline - Through infection & 34.24 & 1.24 & 1.26 \\
\hline - Through vaccination & 49.50 & 65.50 & 65.50 \\
\hline \multicolumn{4}{|c|}{ C) $v=0.1 \%$ population per unit t } \\
\hline HIT was achieved at: & $\mathrm{t}=87$ & $\mathrm{t}=134$ & $\mathrm{t}=107$ \\
\hline$-\mathrm{S}(\mathrm{t})$ & 14.97 & 32.52 & 28.62 \\
\hline$-\mathrm{I}(\mathrm{t})$ & 17.82 & 0.79 & 4.29 \\
\hline - Total infections & 76.33 & 54.08 & 60.68 \\
\hline - Total immunized & 67.21 & 66.70 & 67.09 \\
\hline - Through infection & 58.51 & 53.30 & 56.39 \\
\hline - Through vaccination & 8.70 & 13.40 & 10.70 \\
\hline \multicolumn{4}{|l|}{ At the end of simulation $(\mathrm{t}=200)$} \\
\hline$-\mathrm{S}(\mathrm{t})$ & 6.90 & 32.16 & 27.18 \\
\hline - Total infections & 84.42 & 54.44 & 62.12 \\
\hline - Total immunized & 93.12 & 67.84 & 72.82 \\
\hline - Through infection & 84.42 & 54.44 & 62.12 \\
\hline - Through vaccination & 8.70 & 13.40 & 10.70 \\
\hline
\end{tabular}




\section{Discussion}

Our simulations show that the key to model the transmission dynamics of infectious disease is to allow the transmission rate to decline along with the reducing combined risk of contact infection following recovery. And, this would not alter the early dynamics and basic reproduction number of infectious diseases. However, such modification would lead to very different endings for the same infection as presented in the result section. Infectious diseases modelled by the conventional SIR model appeared to be overly aggressive, and completely impossible to demonstrate control or eradication through herd immunity. This raises a critical concern of using the conventional SIR model or its variants to simulate the ending of the COVID-19 pandemic through herd immunity.

Although Model A successfully demonstrated control and eradication of infectious disease at the level predicted by the HIT, it was not realistic to assume that the transmission rate would decline following infection. Model B provides the framework with a more realistic assumption in modelling the transmission dynamics of infectious diseases with and without vaccination. According to our simulations, the HIT calculated based on the $r_{0}$ of infectious diseases is only accurate if we allow the transmission rate to unrealistically decline following infection with or without vaccination. At lower vaccination rates, the total immunized population contributing to herd immunity may consist of largely individuals immunized through infection, which cannot be predicted using the simple threshold theorem anymore. Therefore, further studies are required to investigate and establish the right threshold for estimating the level of herd immunity.

Importantly, our simulations showed that the transmission rate may decline rapidly after a particular time, depending on the population size, contact rate, $\beta$ and duration of infection. This might help explain the immediate fall of COVID-19 cases in some countries like the United States, United Kingdom and Indonesia, shortly after the rollout of mass and rapid vaccination against the COVID-19 pandemic. Moreover, the newly developed model may provide a better framework for the steady fall of COVID-19 cases in India even without vaccination since September $2020^{24}$. Many researchers attributed the fall of COVID-19 cases without vaccination in India to herd immunity and younger population demographic. A national serological survey conducted by the Indian Council of Medical Research (ICMR) revealed that up to $21 \%$ or 290 million of the adult population in India had developed immunity against the COVID-19 virus ${ }^{25}$.

As of this writing, more than 100 million individuals have been infected by the novel coronavirus with a death toll surpassing $2 \cdot 5$ million $^{24}$. At the same time, many countries have started mass and rapid vaccination with the hope to end the COVID-19 pandemic with herd immunity through vaccination. Therefore, the use of the right modelling frameworks for herd immunity becomes critically important and relevant to support post-vaccination public health planning and preparedness against the pandemic.

\section{Conclusion}

The key to simulating the transmission dynamics of infectious disease with herd immunity is to allow the transmission rate of infectious disease to decline along with the reducing combined 
risk of contact infection following recovery. This can be attained by incorporating the inverse of proportion of recovered individuals into the force of infection of a compartmental model. Further studies are required to establish the right threshold for herd immunity in a randomly mixed population.

\section{References}

1. Jones, D. \& Helmreich, S. A history of herd immunity. The Lancet 396, 810-811 (2020).

2. Fine, P., Eames, K. \& Heymann, D. L. "Herd Immunity": A Rough Guide. Clin. Infect. Dis. 52, 911-916 (2011).

3. Chowell, G., Sattenspiel, L., Bansal, S. \& Viboud, C. Mathematical models to characterize early epidemic growth: A review. Phys. Life Rev. 18, 66-97 (2016).

4. Peak, C. M., Childs, L. M., Grad, Y. H. \& Buckee, C. O. Comparing nonpharmaceutical interventions for containing emerging epidemics. Proc. Natl. Acad. Sci. 114, 4023-4028 (2017).

5. Mandal, S., Sarkar, R. R. \& Sinha, S. Mathematical models of malaria--a review. Malar. J. 10, 202-202 (2011).

6. Law, K. B. et al. Tracking the early depleting transmission dynamics of COVID-19 with a time-varying SIR model. Sci. Rep. 10, 21721 (2020).

7. Keeling, M. J. \& Danon, L. Mathematical modelling of infectious diseases. Br. Med. Bull. 92, 33-42 (2009).

8. Metcalf, C. J. E., Ferrari, M., Graham, A. L. \& Grenfell, B. T. Understanding Herd Immunity. Trends Immunol. 36, 753-755 (2015).

9. Kermack, W. O., McKendrick, A. G. \& Walker, G. T. A contribution to the mathematical theory of epidemics. Proc. R. Soc. Lond. Ser. Contain. Pap. Math. Phys. Character 115, 700721 (1927).

10. Fontanet, A. \& Cauchemez, S. COVID-19 herd immunity: where are we? Nat. Rev. Immunol. 20, 583-584 (2020).

11. Kwok, K. O., Lai, F., Wei, W. I., Wong, S. Y. S. \& Tang, J. W. T. Herd immunity estimating the level required to halt the COVID-19 epidemics in affected countries. J. Infect. 80, e32-e33 (2020).

12. Hwang, J., Park, H., Kim, S.-H., Jung, J. \& Kim, N. Basic and effective reproduction numbers of COVID-19 cases in South Korea excluding Sincheonji cases. medRxiv 2020.03.19.20039347 (2020) doi:10.1101/2020.03.19.20039347.

13. Joseph T, W., Kathy, L. \& Gabriel, L. Nowcasting and forecasting the potential domestic and international spread of the 2019-nCoV outbreak originating in Wuhan, China: a modelling study. The Lancet (2020).

14. Zhuang, Z. et al. Preliminary estimating the reproduction number of the coronavirus disease (COVID-19) outbreak in Republic of Korea and Italy by 5 March 2020. Int. J. Infect. Dis. doi:10.1016/j.ijid.2020.04.044.

15. Zhao, S. et al. Preliminary estimation of the basic reproduction number of novel coronavirus (2019-nCoV) in China, from 2019 to 2020: A data-driven analysis in the early phase of the outbreak. bioRxiv 2020.01.23.916395 (2020) doi:10.1101/2020.01.23.916395.

16. Bartsch, S. M. et al. Vaccine Efficacy Needed for a COVID-19 Coronavirus Vaccine to Prevent or Stop an Epidemic as the Sole Intervention. Am. J. Prev. Med. 59, 493-503 (2020). 
17. Bubar, K. M. et al. Model-informed COVID-19 vaccine prioritization strategies by age and serostatus. Science eabe6959 (2021) doi:10.1126/science.abe6959.

18. Sandmann, F., Davies, N., Vassall, A., Edmunds, W. J. \& Jit, M. The potential health and economic value of SARS-CoV-2 vaccination alongside physical distancing in the UK: transmission model-based future scenario analysis and economic evaluation. medRxiv 2020.09.24.20200857 (2020) doi:10.1101/2020.09.24.20200857.

19. Scherer, A. \& McLean, A. Mathematical models of vaccination. Br. Med. Bull. 62, 187199 (2002).

20. Wilson, E. \& Worcester, J. The Law of Mass Action in Epidemiology. Proc. Natl. Acad. Sci. U. S. A. 31, 24-34 (1945).

21. Heffernan, J. M., Smith, R. J. \& Wahl, L. M. Perspectives on the basic reproductive ratio. J. R. Soc. Interface 2, 281-293 (2005).

22. Siettos, C. I. \& Russo, L. Mathematical modeling of infectious disease dynamics. Virulence 4, 295-306 (2013).

23. Dietz K. Transmission and control of arbovirus diseases. In: Ludwig D, Cooke KL, eds. Epidemiology. Pheladelphia PA: Society for Industrial and Applied Mathematics, 1975: 10421.

24. Johns Hopkins University Coronavirus Resources Center. https://coronavirus.jhu.edu/.

25. ICMR sero survey: One in five Indians exposed to Covid-19. BBC News (2021).

Acknowledgment

We would like to thank the Director General of Health Malaysia for his permission to publish this article. We would like to thank Associate Professor Dr. Jane Labadin and Dr. Kuan Pei Xuan for proofreading the article and providing constructive inputs before publication.

\section{Competing interests}

The authors declare no competing interests.

\section{Author information}

Digital Health Research and Innovation Unit, Institute for Clinical Research, National Institutes of Health, Ministry of Health Malaysia

Kian Boon Law

\section{Institute for Clinical Research, National Institutes of Health, Ministry of Health Malaysia Kalaiarasu M. Peariasamy}

\section{Office of the Director General, Ministry of Health Malaysia}

Noor Hisham Abdullah \& Hishamshah Ibrahim

\section{Author Contributions}

K.B.L and K.M.P conceived and planned the study.

K.B.L contributed to the design of the compartmental model and simulation.

K.M.P, H.S and N.H.A supervised the implementation of the study.

Data analysis and graphics were done by K.B.L.

All authors contributed to the interpretation of the findings and writing of article and approved the final version for publication. 
559 Corresponding author

560 Correspondence to Kian Boon Law

561

562 Ethics requirement

563 The study was registered with National Medical Research Register. No ethics approval was 564 required. 
Figures

A) Compartmental structure

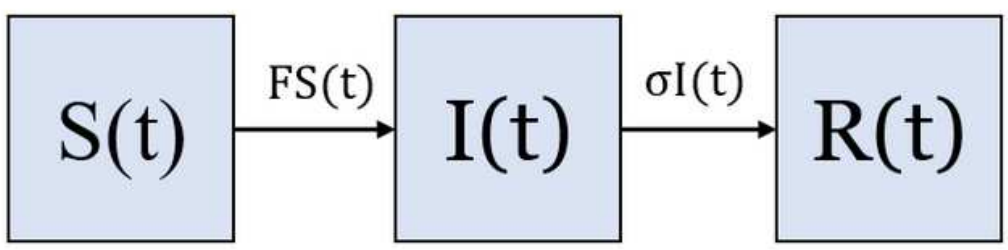

B) Model equations

$$
\begin{aligned}
& S(t)=-F S(t) \\
& I(t)=F S(t)-\sigma I(t) \\
& R(t)=\sigma I(t)
\end{aligned}
$$

Force of infection:

$$
F=\beta \frac{I(t)}{N}
$$

\section{Figure 1}

The compartmental structure and model equations of Kermack \& Mckendrick's SIR model.

\section{A) Compartmental structure}

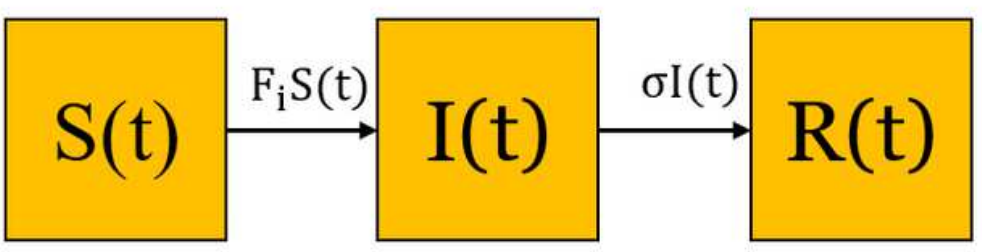

B) Model equations

$$
\begin{aligned}
& S(t)=-F_{i} S(t) \\
& I(t)=F_{i} S(t)-\sigma I(t) \\
& R(t)=\sigma I(t)
\end{aligned}
$$

\section{Force of infection:}

$$
\begin{aligned}
& \text { Model A: } F_{i=A}=\beta \frac{I(t)[S(t)]^{2}}{N^{2}} \\
& \text { Model B: } F_{i=B}=\beta \frac{I(t)[N-R(t)]}{N^{2}}
\end{aligned}
$$

\section{Figure 2}

The compartmental structure and model equations of the newly developed Model A and Model B. 
A) Compartmental structure

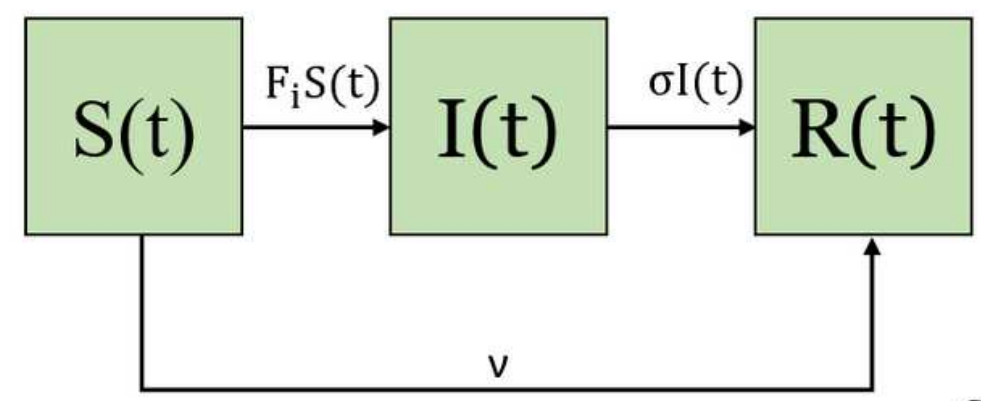

Vaccination at rate, $v$
B) Model equations

$$
\begin{aligned}
& \mathrm{S}(\mathrm{t})=-\mathrm{F}_{\mathrm{i}} \mathrm{S}(\mathrm{t})-v \\
& \mathrm{I}(\mathrm{t})=\mathrm{F}_{\mathrm{i}} \mathrm{S}(\mathrm{t})-\sigma \mathrm{I}(\mathrm{t}) \\
& \mathrm{R}(\mathrm{t})=\sigma \mathrm{I}(\mathrm{t})+v
\end{aligned}
$$

\section{Force of infection}

Conventional model: $F=\beta \frac{I(t)}{N}$

$$
\begin{aligned}
& \text { Model A: } F_{i=A}=\beta \frac{I(t)[S(t)]^{2}}{N^{2}} \\
& \text { Model B: } F_{i=B}=\beta \frac{I(t)[N-R(t)]}{N^{2}}
\end{aligned}
$$

\section{Figure 3}

The compartmental structure and model equations of vaccine models modified using the conventional SIR model, Model A and Model B.

A) Conventional SIR model

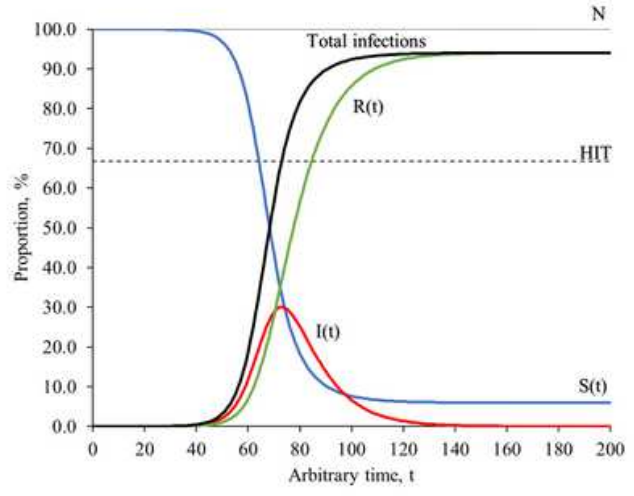

D) Conventional SIR model versus Model A

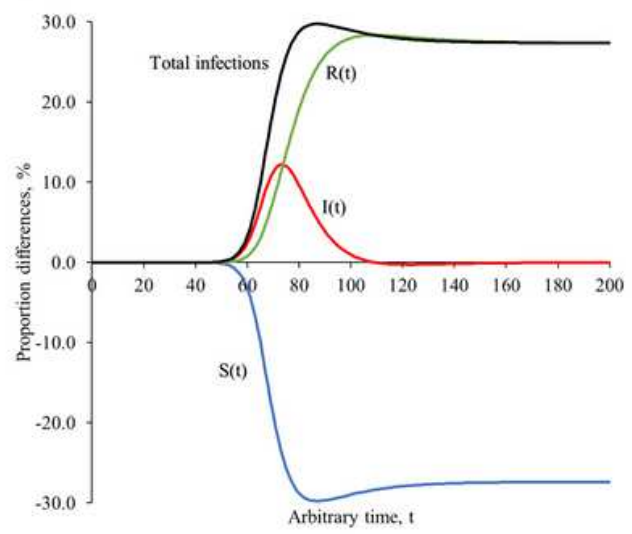

B) Model A

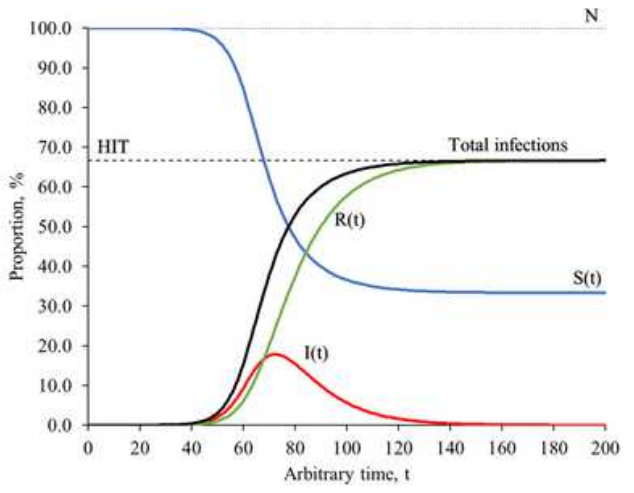

E) Conventional SIR model versus Model B

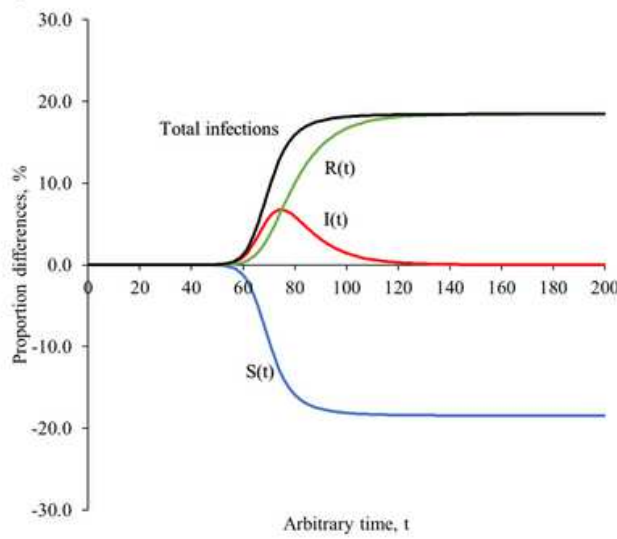

C) Model B

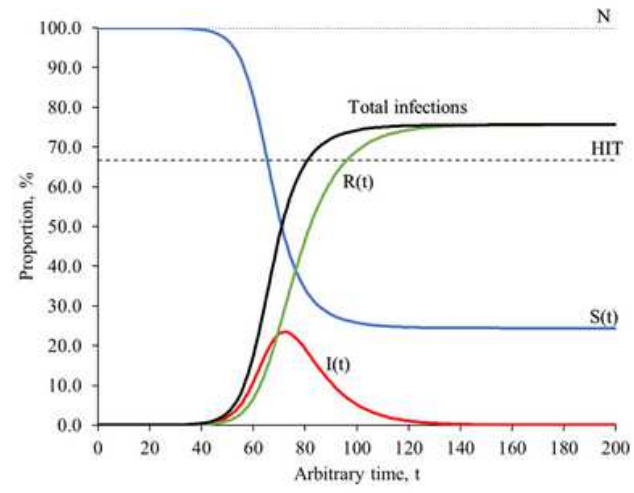

F) Total infections with varying $r_{0}$

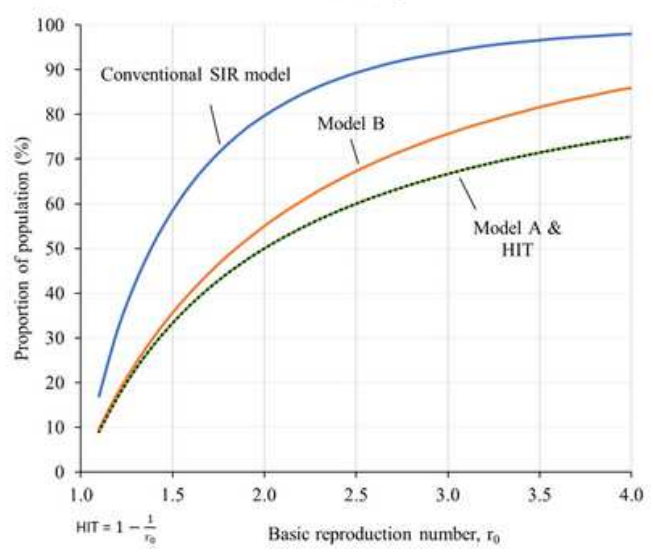


Figure 4

Transmission dynamics of infectious diseases with herd immunity through infection Part A, B and C presents transmission dynamics of infectious diseases with herd immunity through infection simulated by the conventional SIR model, Model A and Model B. Part D and E presents proportion differences between the conventional SIR model and Model A and between the conventional SIR mode and Model B. Part F presents total infections generated by the conventional SIR model, Model A and Model B with varying $\mathrm{r} 0$.

A) Conventional SIR ( $v=1.0 \%$ )

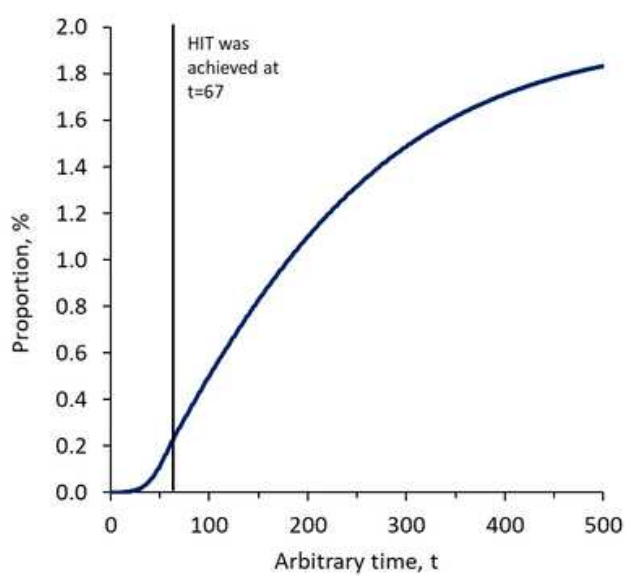

D) Conventional SIR ( $v=0.5 \%$ )

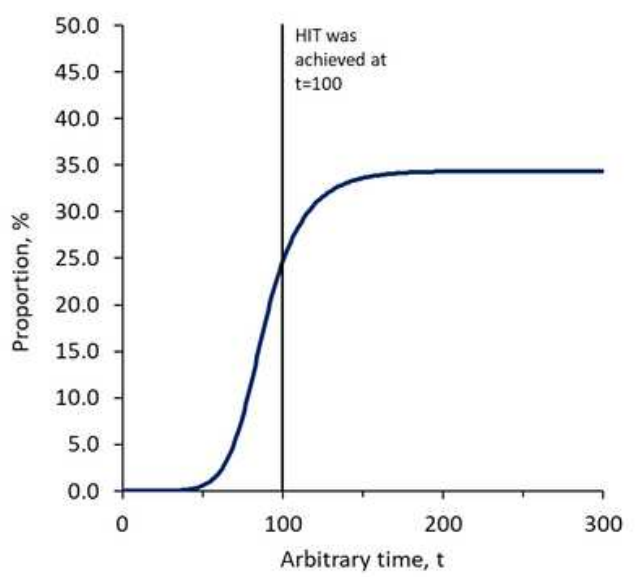

G) Conventional SIR ( $v=0.1 \%$ )

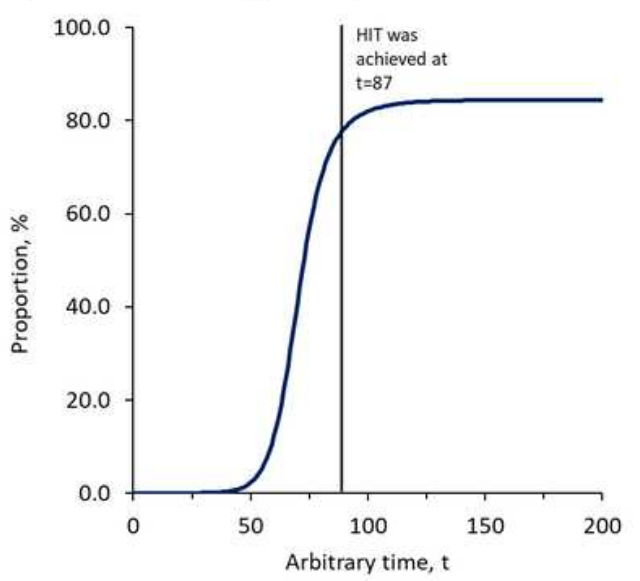

B) Model A ( $v=1.0 \%)$

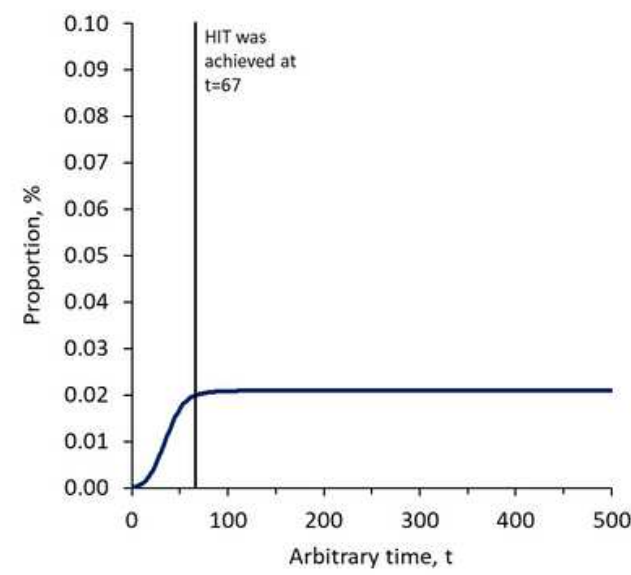

E) Model $A(v=0.5 \%)$

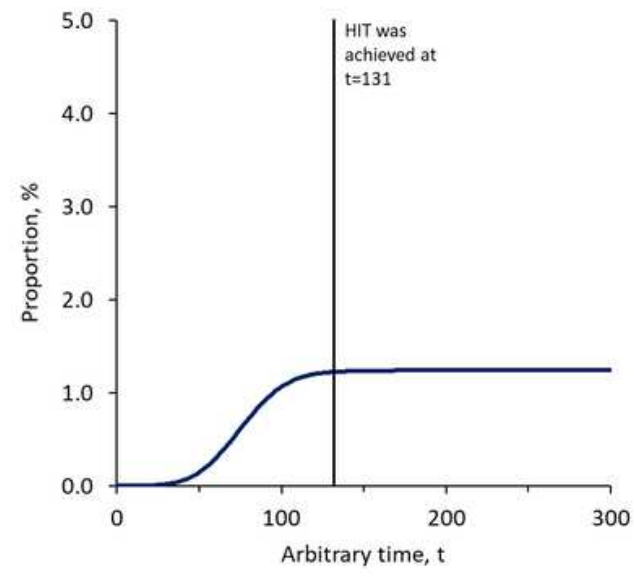

H) Model A ( $v=0.1 \%)$

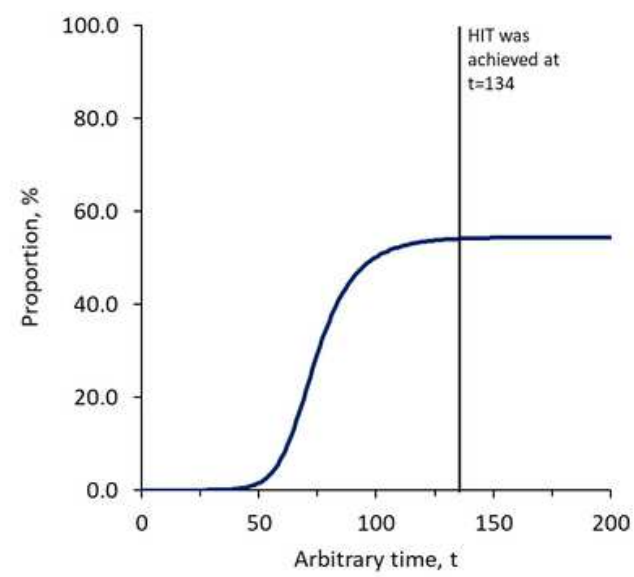

C) Model B ( $v=1.0 \%)$

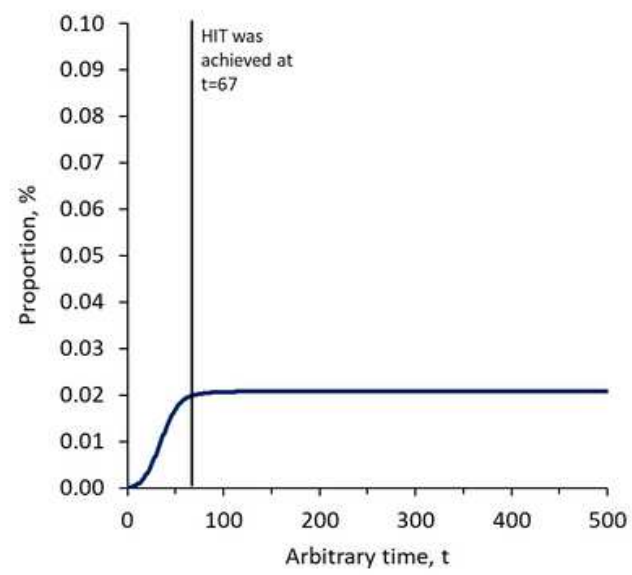

F) Model $B(v=0.5 \%)$

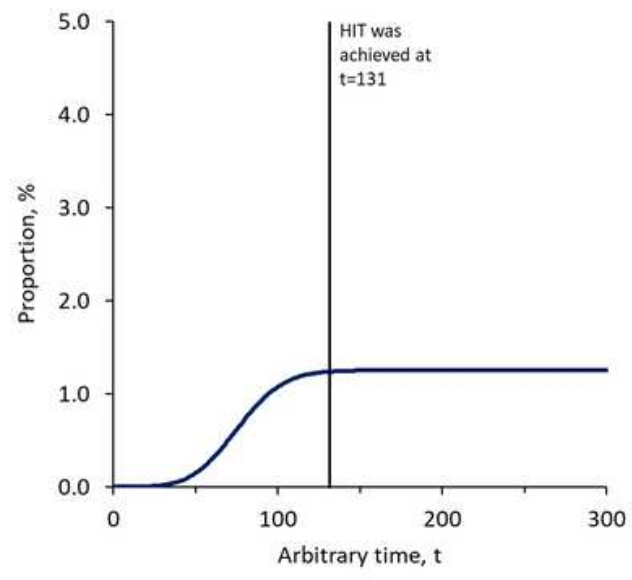

I) Model $B(v=0.1 \%)$

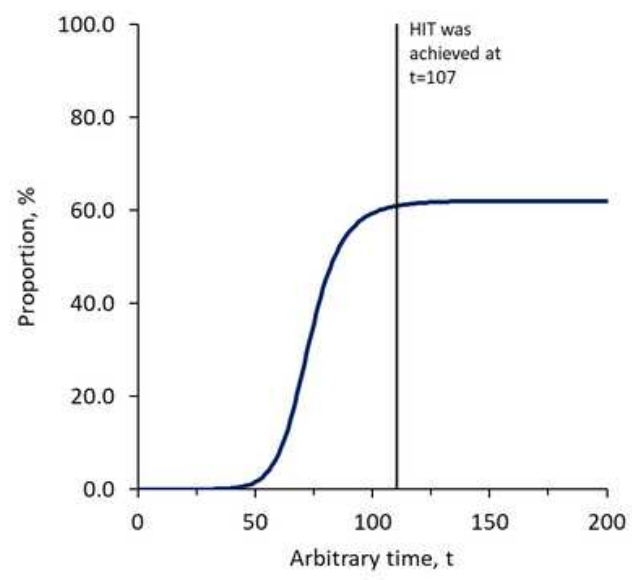




\section{Figure 5}

Transmission dynamics of infectious diseases with herd immunity through vaccination Part $A, B$ and $C$ present transmission dynamics of infectious diseases at vaccination rate, $v=1.0 \%$ population per unit $t$ projected by the conventional SIR model, Model A and Model B. The HIT was reached at $t=67$ in all three models. Part D, E and F present transmission dynamics of infectious diseases at $\mathrm{v}=0.5 \%$ population per unit t projected by the conventional SIR model, Model A and Model B. The HIT was reached at $t=100$ in the conventional SIR model, and at $\mathrm{t}=131$ in both Model A and Model B. Part G, H and I present transmission dynamics of infectious disease at $\mathrm{v}=0.1 \%$ population per unit $\mathrm{t}$ projected by the conventional SIR model, Model A and Model B. The HIT was reached at $t=87$ in the conventional SIR model, at $t=134$ in Model A, and t=107 in Model B. Times to reach the HIT was marked by vertical lines. 